\title{
Particle detectors for the future antiproton Paul trap of the ASACUSA experiment at CERN
}

\author{
A. Soter · M. Hori
}

Published online: 26 August 2009

(C) The Author(s) 2009. This article is published with open access at Springerlink.com

\begin{abstract}
Two detectors which will be used to commission a superconducting radiofrequency Paul trap for antiprotons, now being constructed at CERN and MPQ, are described. One is a microwire secondary electron emission monitor which will nondestructively measure the spatial profile of a low energy $(E=10-100 \mathrm{keV})$ antiproton beam. The other is a system of electromagnetic shower counters which will detect the secondary particles emerging from the antiproton annihilations occurring in the trap.
\end{abstract}

Keywords Paul trap · Microwire detector $\cdot$ Shower counter

\section{Introduction}

The ASACUSA collaboration at CERN and the Max Planck Institute of Quantum Optics are now constructing a superconducting radiofrequency Paul trap for antiprotons. In this paper we describe two particle detectors [1-4], which will be used to commission this trap. In the proposed experiment, a radiofrequency quadrupole decelerator (RFQD) will first reduce the energy of the antiprotons ejected from CERN's Antiproton Decelerator (AD) from E=5.3 MeV to 50-100 keV. These

A. Soter $(\bowtie)$

KFKI Research Institute for Particle and Nuclear Physics,

Konkoly T. M. 29-33, 1121 Budapest, Hungary

e-mail: Anna.Soter@cern.ch

A. Soter · M. Hori

Max-Planck-Institut für Quantenoptik,

Hans-Kopfermann-Str. 1, 85748 Garching, Germany

M. Hori

Department of Physics, University of Tokyo,

7-3-1 Hongo, Bunkyo-ku, Tokyo 113-0033, Japan 
antiprotons will then be injected into the trap. It is essential to accurately monitor the spatial profile of this beam, to ensure a high trapping efficiency.

We constructed a microwire secondary electron emission chamber $[1,2]$ for this purpose. It allowed $98-99 \%$ of the antiprotons to pass through without any degradation, whereas a small fraction (1-2\%) was intercepted by a series of wire grids and produced [5] the signal.

The time evolution of the number of antiproton annihilations that occur in the trap will be measured by electromagnetic shower counters [6-9] surrounding the trap. We carried out Monte-Carlo simulations using the GEANT4 package [10] to optimize their detection efficiency.

\section{Microwire secondary electron emission detector}

The antiproton beam profile monitor $[1,2]$ has been used in recent laser spectroscopy experiments on antiprotonic helium atoms [11-13] and ions [14]. It has a spatial resolution $\Delta x=1 \mathrm{~mm}$ and active area of $32 \times 32 \mathrm{~mm}$. Grid electrodes arranged in a XY configuration, consisting of 5-10- $\mu m$-diameter wires, were suspended in an ultra-high vacuum chamber (Fig. 1a and b). The AD delivered a 100-ns-long pulsed beam containing $N=3 \cdot 10^{7}$ antiprotons at a repetition rate $f=0.01 \mathrm{~Hz}$. A small percentage $(1 \%)$ of the antiprotons were intercepted by the wires and induced the emission [5] of a few thousand secondary electrons. Charge-sensitive preamplifiers measured the charge ejected from each of the 64 wires on the X and Y photocathode grids (Fig. 2a). The monitor was operated at cryogenic temperatures $(T<100 \mathrm{~K})$ and high magnetic fields $(B>0.1 \mathrm{~T})$.

To maximize the sensitivity [15], junction field-effect transistors with a low $1 / \mathrm{f}$ noise coefficient $K_{f} \sim 10^{-27} \mathrm{~J}$ and input capacitance $C_{a m p} \sim 10 \mathrm{pF}$, a high transconductance $g_{m} \sim 50 \mathrm{mS}$ and feedback resistance $R_{f}=5 \mathrm{G} \Omega$ were used in the preamplifiers (Fig. 2a). A transconductance amplifier converted the voltage signal into a differential current one with a conversion ratio of $d I / d V=0.03 \mathrm{~A} / \mathrm{V}$. The signals were then transmitted from the preamplifiers to active filter amplifiers over several meters of shielded twisted-pair cable. This differential nature and low impedance of the signal ensured that any external electromagnetic interference picked up on the cable would not degrade the signal-to-noise ratio. This was important because the monitor was used next to the RFQD driven at frequency $f \sim 200 \mathrm{MHz}$. The detector had an equivalent noise charge [15] of $\varepsilon \sim 200$ electron. This is within factor 2 of the best values reported for any room temperature detector of comparable $10 \mathrm{pF}$ capacitance.

The monitor was also sensitive to a pulsed Nd:YAG laser beam of wavelength $\lambda=$ $213 \mathrm{~nm}$ and energy $E \sim 5 \mathrm{pJ}$. The measured quantum efficiency $\gamma=8 \cdot 10^{-5}$ agreed within a factor of 2 with experimental values [16]. At longer wavelengths $\lambda=355 \mathrm{~nm}$ and $532 \mathrm{~nm}$, the electron emission could only proceed via the nonlinear, simultaneous absorption of several photons.

We measured the spatial profiles of a pulsed beam containing $2 \cdot 10^{6}$ antiprotons (Fig. 2b, solid lines) of energy $E=60 \mathrm{keV}$, and a laser beam of wavelength $\lambda=$ $289 \mathrm{~nm}$ (broken lines). The two beams were fired collinearly into the wire monitor. The antiproton beam was found to consist of a dense core of diameter $d_{c}=10 \mathrm{~mm}$ 

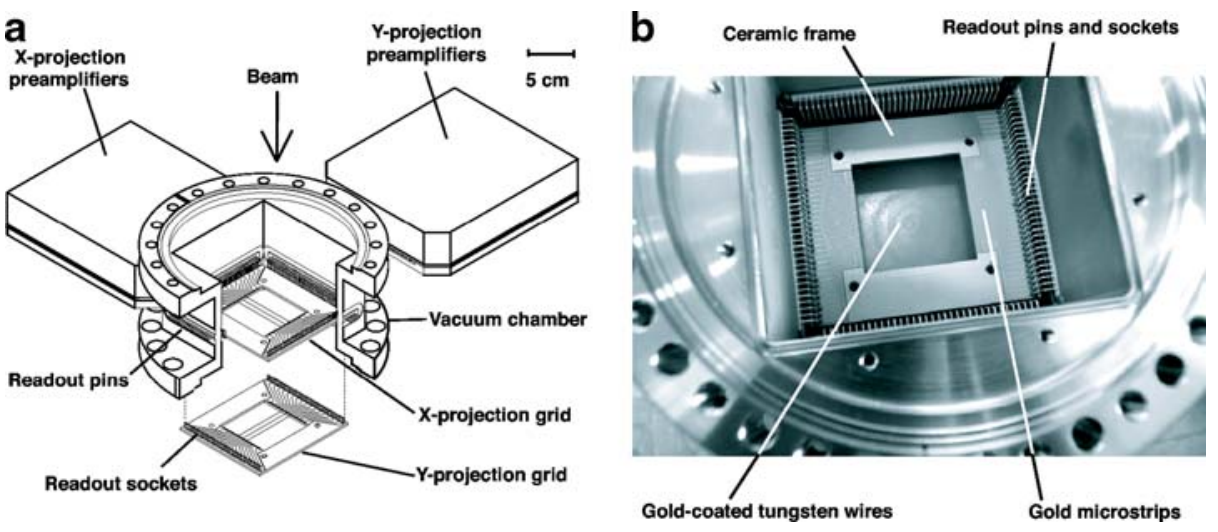

Fig. 1 Schematic drawing of the microwire secondary electron emission detector (a), and photograph of its interior with a grid installed $(\mathbf{b})$

Fig. 2 Layout of the readout eletronics of the microwire monitor (a), and the measured spatial profiles of a pulsed beam containing $2 \cdot 10^{6}$ antiprotons (solid lines), and a laser beam of wavelength $\lambda=289 \mathrm{~nm}$ (broken lines)
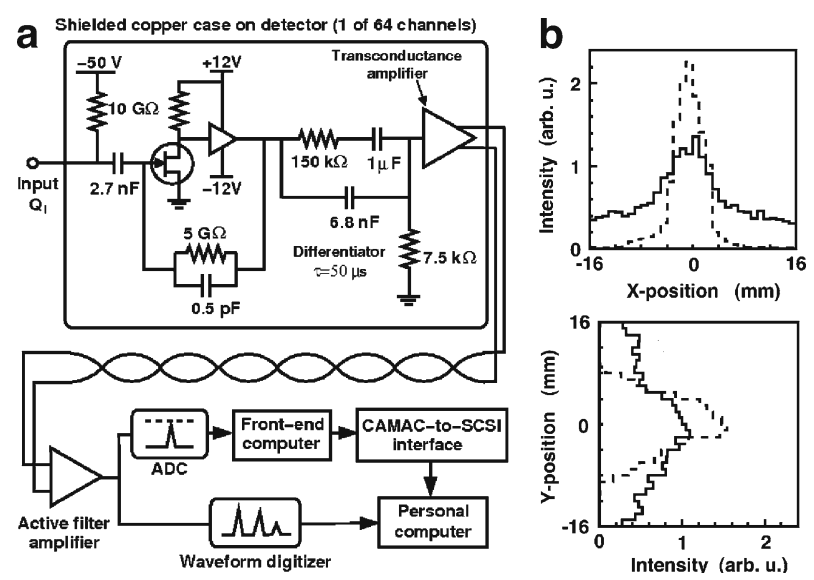

containing half of the antiprotons, and a halo of diameter $d_{h}=30 \mathrm{~mm}$ containing the rest. Each wire intercepted between a few hundred and a few thousand antiprotons.

\section{Electromagnetic shower counters}

We intend to construct ten electromagnetic shower counters of typical size $400 \mathrm{~mm} \times$ $500 \mathrm{~mm}$ (Fig. 3). These will be positioned to cover a solid angle of $2 \pi$ steradians seen from the trap. Each counter contains 2-3 lead and plastic scintillator layers arranged in a sandwich structure.

In the Monte Carlo simulation, we used the branching ratios for antiproton-proton and antiproton-neutron reactions measured in various experiments [3]. From this, the momentum distributions and multiplicities of secondary pions emerging from antiproton annihilations on helium were calculated. These results agreed with other experimental data within $20 \%$ [17]. 
Fig. 3 Schematic drawing of the interior of a shower counter

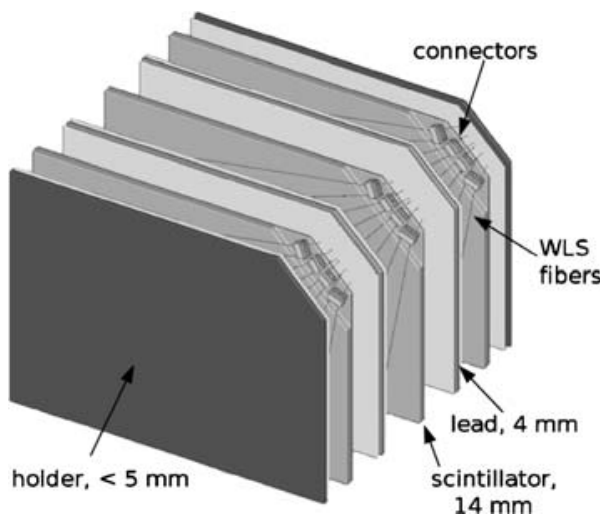

The thickness, layer numbers, and sizes of the detectors were varied in the simulation, under the requirement of 2-3 secondary particles striking the counters in coincidence. The results indicated that, i) the efficiency for detecting 2- and 3 -particle coincidence events are $95 \%$ and $56 \%$, respectively ii) the detection efficiency is maximized when the thickness of the lead layers are slightly less than its radiation length $(5.6 \mathrm{~mm})$, iii) the optimum thicknesses and layer numbers of the scintillator are $10-14 \mathrm{~mm}$ and 3 , respectively.

This design of high occupancy can only be achieved by using a compact way of reading out the counters. For this, we intend to embed 1-mm-diameter, Y11 wavelength-shifting fibers in grooves cut into the scintillators. When a minimumionizing particle hits the counter, some 100 fluorescence photons are emitted from the ends of each fiber [6]. These photons will be detected either by conventional photomultiplier tubes, or 1-mm-diameter, multipixel-photon-counters (MPPC's). We expect to achieve high spatial uniformity response (non-uniformity $<10 \%,[7,8]$ ) and 1-ns-scale timing resolution [9].

Acknowledgements We are deeply indebted to the ASACUSA collaboration, M. Mitani, and K. Mori. This work was supported by the EURYI award of the European Science Foundation and the Deutsche Forschungsgemeinschaft, the Munich Advanced Photonics (MAP) cluster, the MaxPlanck-Gesellschaft, the Global COE Program "the Physical Sciences Frontier" of MEXT, Japan, the Grant-in-Aid for Specially Promoted Research (20002003) of MEXT, Japan, the Mitsubishi Foundation, the Hungarian Science Foundation and the Hungarian National Bureau of Science and Development (Contracts OTKA NK67974, K72172, H07C-74153 and JAP-21/2006).

Open Access This article is distributed under the terms of the Creative Commons Attribution Noncommercial License which permits any noncommercial use, distribution, and reproduction in any medium, provided the original author(s) and source are credited.

\section{References}

1. Hori, M.: Photocathode microwire monitor for nondestructive and higly sensitive spatial profile measurements of ultraviolet, x-ray, and charged particle beams. Rev. Sci. Instrum. 76, 113303-1-6 (2005)

2. Hori, M.: Parallel plate chambers for monitoring the profiles of high-intensity pulsed antiproton beams. Nucl. Instrum. Methods Phys. Res. A 522, 420 (2004) 
3. Hori, M., Yamashita, K., Hayano, R.S., Yamazaki, T.: Analog Cherenkov detectors used in laser spectroscopy experiments on antiprotonic helium. Nucl. Instrum. Methods Phys. Res. A 496, 102 (2003)

4. Hori, M., Hanke, K.: Spatial and temporal beam profile monitor with nanosecond resolution for CERN's linac4 and superconducting proton linac. Nucl. Instrum. Methods. A 588, 359 (2008)

5. Hasselkamp, D., Rothard, H., Groeneveld, K.-O., Kemmler, J., Varga, P., Winter, H.: Particle Induced Electron Emission II. Springer, Heidelberg (1992)

6. Albrow, M.G., et al.: Nucl. Instrum. Methods Phys. Res. A 256, 23 (1987)

7. Artikov, A., et al.: Nucl. Instrum. Methods Phys. Res. A 538, 358 (2005)

8. Aota, S., et al.: Nucl. Instrum. Methods Phys. Res. A 352, 566 (2005)

9. Kudenko, Yu.G., et al.: Nucl. Instrum. Methods Phys. Res. A 469, 340 (2001)

10. Agostinelli, S., et al.: Nucl. Instrum. Methods Phys. Res. A 506, 250 (2003)

11. Hori, M., et al.: Phys. Rev. Lett. 87, 093401 (2001)

12. Hori, M., et al.: Phys. Rev. Lett. 91, 123401 (2003)

13. Hori, M., et al.: Phys. Rev. Lett. 96, 243401 (2006)

14. Hori, M., et al.: Phys. Rev. Lett. 94, 063401 (2005)

15. Radeka, V.: Annu. Rev. Nucl. Part. Sci. 38, 217 (1988)

16. Boscolo, I., et al.: J. Appl. Phys. 87, 4005 (2000)

17. Bendiscioli, G., et al.: Nucl. Phys. A 518, 683-708 (1990) 